\title{
Non-Faradaic electric currents in the Nernst-Planck equations and nonlocal diffusiophoresis of suspended colloids in crossed salt gradients
}

\author{
Patrick B. Warren
}

\section{Published version information}

Citation: PB Warren. "Non-Faradaic electric currents in the Nernst-Planck equations and nonlocal diffusiophoresis of suspended colloids in crossed salt gradients." Physical Review Letters, vol. 124, no. 24 (2020): 248004.

\section{DOI: $10.1103 /$ PhysRevLett.124.248004}

This version is made available in accordance with publisher policies. Please cite only the published version using the reference above. This is the citation assigned by the publisher at the time of issuing the APV. Please check the publisher's website for any updates. 


\title{
Non-Faradaic Electric Currents in the Nernst-Planck Equations and Nonlocal Diffusiophoresis of Suspended Colloids in Crossed Salt Gradients
}

\author{
Patrick B. Warren $\oplus^{*}$ \\ STFC Hartree Centre, Scitech Daresbury, Warrington WA4 4AD, United Kingdom \\ and Unilever R\&D Port Sunlight, Quarry Road East, Bebington, Wirral CH63 3JW, United Kingdom
}

(Received 11 December 2019; accepted 22 May 2020; published 16 June 2020)

\begin{abstract}
In the Nernst-Planck equations in two or more dimensions, a non-Faradaic solenoidal current can arise as a consequence of connecting patches with different liquid junction potentials. Whereas this current vanishes for binary electrolytes or in one-dimensional problems, it is in general nonvanishing, for example, in crossed salt gradients. For a suspended colloidal particle, chemiphoresis in the concentration gradients is generally vectorially misaligned with electrophoresis in the electrostatic potential gradient, and there is a nonlocal contribution to the latter deriving from the Ohmic electric field associated with the current; in a case study this contributes up to $20 \%-30 \%$ of the total effect.
\end{abstract}

DOI: 10.1103/PhysRevLett.124.248004

The growing realization that diffusiophoresis is a potent and ubiquitous nonequilibrium transport mechanism for micron-sized colloidal particles has led to a recent surge of interest in the phenomenon [1-12]. For example, diffusiophoresis is effective at injecting or ousting particles from dead-end channels $[13,14]$, has been identified as a hitherto unsuspected pore-scale particulate soil removal process in laundry detergency [15], and can be used to manipulate and sort particles by size and charge [14,16,17]. The biggest effects arise in electrolyte solutions, where chemiphoresis in concentration gradients combines with electrophoresis in the diffusion potential to drive particles at speeds of 1-10 $\mu \mathrm{m} \mathrm{s}^{-1}$ [18], propelling them over large distances in timescales of minutes. An additional peculiarity in binary electrolytes is that the speed is logarithmically dependent on the concentration, leading to persistent effects such as osmotic trapping [2] and long-lived particle removal [15].

To my knowledge, the existing phenomena that have been discussed in the above context pertain to binary electrolytes or assume one-dimensional gradients [6,1923]. In this Letter, I argue that a still further enriched phenomenology arises in multicomponent electrolytes when concentration gradients are superimposed in different directions ("crossed" salt gradients). In part this is because chemiphoresis decouples partially from electrophoresis, but additionally it is because a nonvanishing electric current arises even in the absence of Faradaic reactions, when patches with different liquid junction potentials are connected by the intervening electrolyte solution. In itself this is surely a fascinating phenomenon, but importantly for diffusiophoresis the nonvanishing current means there is a nonlocal contribution to diffusiophoresis that can only be elucidated by solving the whole ion transport problem. Since it seems quite possible to engineer crossed gradients with dissolving salt crystals [3], ion-exchange resins [5], suitably chosen "soluto-inertial beacons" [7,24], or by using $\mathrm{CO}_{2}$ to generate carbonic acid gradients [10], and perhaps in combination with merging streams with different salinities [1], these observations may provide novel opportunities for particle manipulation and sorting.

Let me start with the Nernst-Planck equations which govern ion transport in these problems [25,26],

$$
\frac{\partial \rho_{i}}{\partial t}+\nabla \cdot \mathbf{J}_{i}=0, \quad \mathbf{J}_{i}=-D_{i}\left(\nabla \rho_{i}+\rho_{i} z_{i} \nabla \varphi\right)
$$

In these, $\rho_{i}$ is the density of the $i$ th ionic species, $D_{i}$ is the corresponding diffusion coefficient, $z_{i}$ the charge on the ion in units of $e$, where $e$ is the unit of elementary charge, and $\varphi=e \phi / k_{\mathrm{B}} T$ is a dimensionless electrostatic potential wherein $k_{\mathrm{B}} T$ is the unit of thermal energy and $\phi$ is the actual electrostatic potential. Equation (1) combines mass conservation laws for the individual ion densities with expressions for the fluxes driven by diffusion and drift in the electric field. For simplicity I omit advection terms although these are certainly relevant in microfluidics devices, and may additionally arise if bulk flows are driven by diffusio-osmotic effects (see further below) [14].

The Nernst-Planck equations must be augmented by a closure for the electrostatic potential. At a fundamental level this is the Poisson equation, $\epsilon \nabla^{2} \phi=-e \rho_{z}$, where $\rho_{z}=\sum_{i} z_{i} \rho_{i}$ is the space charge (in units of $e$ ) and $\epsilon$ is the permittivity (assumed constant) of the supporting medium. The combined set are then known as the Poisson-NernstPlanck (PNP) equations. Introducing the Debye length $\lambda_{D}=\left(\epsilon k_{\mathrm{B}} T / e^{2} \rho_{D}\right)^{1 / 2}$, where $\rho_{D}=\sum_{i} z_{i}^{2} \rho_{i}$, allows the Poisson equation to be written as $\lambda_{D}^{2} \nabla^{2} \varphi=-\rho_{z} / \rho_{D}$. This makes it clear that if the problem size $L \gg \lambda_{D}$, the bare electrostatics problem is singular [27-32], in the sense that there is an "outer" domain on the length scale $O(L)$ in 

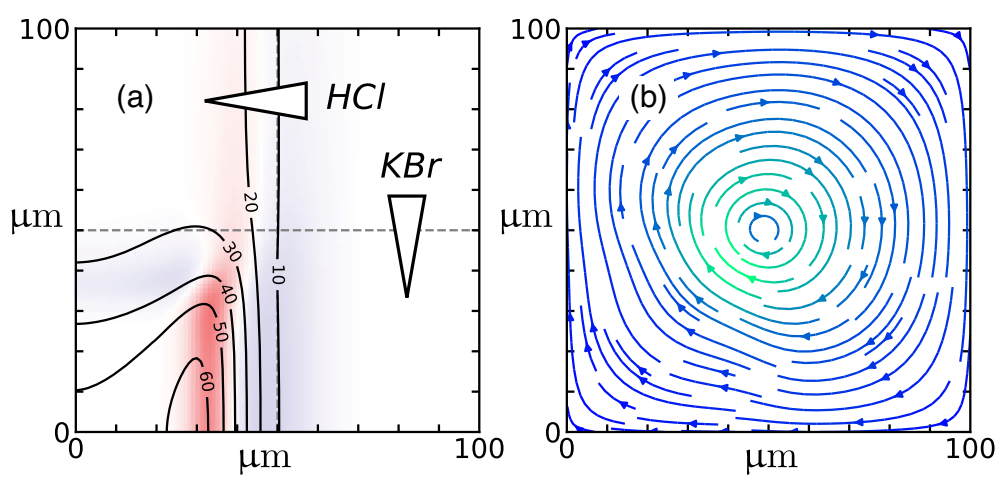

(c)
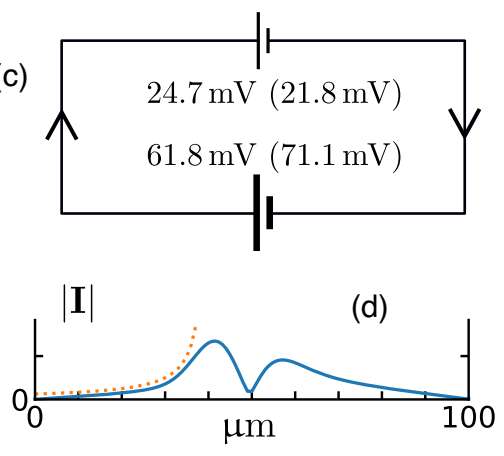

FIG. 1. Crossed salt gradients between $\mathrm{HCl}$ (horizontal) and $\mathrm{KBr}$ (vertical), localized initially to midplanes [41]. (a) Electrostatic potential (equipotential lines labeled in $\mathrm{mV}$ ) and space charge (colored background). (b) Circulating electric current (colored by magnitude). (c) Equivalent circuit labeled by the $\mathrm{HCl}$ liquid junction potentials (in brackets are the "open circuit" values calculated from $\Delta \varphi=-\beta \ln \left(\sigma_{2} / \sigma_{1}\right)$ where $\beta \approx 0.64$ and $\sigma_{2} / \sigma_{1}$ is the ratio of conductivities across the junction [36]). (d) Magnitude of current along the $x=y$ diagonal (the dotted line is $1 / r \sim 1 /\left|x-x_{0}\right|$ where $x_{0}=40 \mu \mathrm{m}$ ).

which $\rho_{z} \approx 0$ (local charge neutrality), asymptotically matched to "inner" solutions on a length scale $O\left(\lambda_{D}\right)$ (i.e., electric double layers or EDLs), whenever the boundary conditions would otherwise overdetermine $\varphi$ in the outer domain [33].

Crucially, local charge neutrality does not necessarily imply a vanishing electric current $\mathbf{I}=\sum_{i} z_{i} \mathbf{J}_{i}$ (in units of $e$ ) in the outer domain. Rather, by summing the mass conservation laws in Eq. (1), one can only conclude that the current should be solenoidal $(\nabla \cdot \mathbf{I}=0)$. In fact, even for pure diffusion problems without Faradaic reactions [34], a nonvanishing current $(\mathbf{I} \neq 0)$ is not only possible but may be mandatory. To see this, insert the fluxes $\mathbf{J}_{i}$ from Eq. (1) into the definition of I to obtain [26]

$$
\mathbf{I}=-\nabla g+\sigma \mathbf{E}
$$

where $g=\sum_{i} z_{i} D_{i} \rho_{i}$ is a weighted sum of ion densities, $\sigma=\sum_{i} z_{i}^{2} D_{i} \rho_{i}$ is the conductivity, and $\mathbf{E}=-\nabla \varphi$ is the electric field (the latter two are in semireduced units). The two terms in Eq. (2) correspond to a diffusion current, and a conduction current obeying Ohm's law. By taking the curl of Eq. (2) one can eliminate the electrostatic potential to find

$$
\sigma \nabla \times \mathbf{I}=\nabla \sigma \times(\mathbf{I}+\nabla g) .
$$

This is an inhomogeneous partial differential equation for $\mathbf{I}$ in which the source term $\nabla \sigma \times \nabla g$ mandates $\mathbf{I} \neq 0$ [35].

Of course there are many examples where I does vanish. One such case is where the gradients are one dimensional so that $\varphi$ can be found by quadrature [21]. Another important case is that of a binary electrolyte $[25,26]$ for which $\varphi=-\beta \ln \rho_{s}$ (the diffusion or liquid junction potential). Here $\beta=\left(D_{1}-D_{2}\right)\left(q_{1} D_{1}+q_{2} D_{2}\right)$ is a normalised diffusivity contrast, and I suppose that $z_{1}>0$ and $z_{2}<0$, set $q_{i}=\left|z_{i}\right|$, and use $\rho_{s}=\rho_{1} / q_{2}=\rho_{2} / q_{1}$ for the overall electrolyte concentration [36].

To summarize the mathematical problem thus far, given $g$ and $\sigma$ and supposing that $\mathbf{I} \cdot \mathbf{n}$ is specified on the boundaries of the domain of interest, we must find the current distribution that satisfies Eq. (2) with $\nabla \cdot \mathbf{I}=0$ and $\mathbf{E}=-\nabla \varphi$. To prove solutions do exist, and are unique, we can note that this combination implies [26,37]

$$
\nabla(\sigma \nabla \varphi)+\nabla^{2} g=0 .
$$

This is an inhomogeneous Poisson equation for $\varphi$ with the equivalent of a spatially varying dielectric permittivity. Existence and uniqueness of $\varphi$ (up to an additive constant) then follows by analogy with standard electrostatics [38]. A direct proof is given in the Supplemental Material [39], along with a discussion of an equivalent variational principle. Equation (4) is nonsingular and amenable to solution by standard numerical methods, and replaces the original electrostatic Poisson equation in closing the Nernst-Planck equations.

Let me turn now to a numerical case study which demonstrates the principles by which a nonvanishing electric current arises. To distinguish between the crossed gradients, I set the problem up with four rather than three ions, which would be the minimal example of a crossed salt gradient. I consider a $100 \mu \mathrm{m}$ square domain in which a 100 -fold gradient in an electrolyte with a large diffusivity contrast $(\mathrm{HCl}, \beta \approx 0.64)$ is crossed with a 100 -fold gradient in a supporting electrolyte $(\mathrm{KBr}, \beta \approx-0.01)$ [40]. The concentration gradients are initially localized to the midplanes, with widths $10 \mu \mathrm{m}$ [41], dividing the square domain into four quadrants as shown in Fig. 1(a). The actual concentration units need not be specified since the overall units of concentration can be factored out of the NernstPlanck equations. 

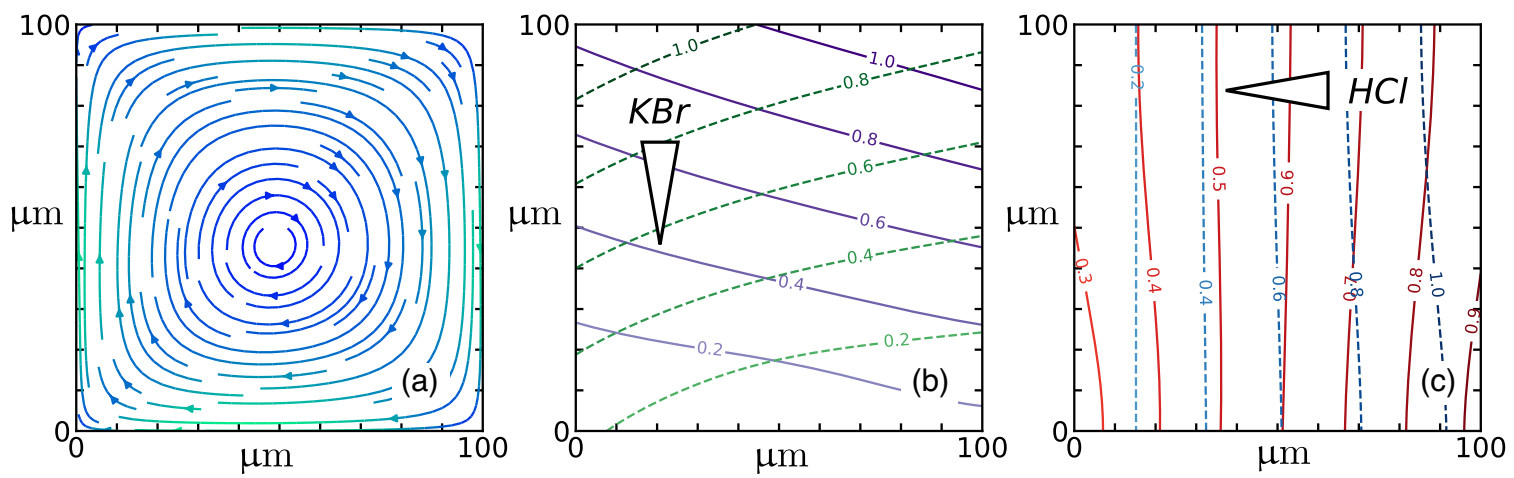

FIG. 2. Steady-state crossed salt gradients. (a) Circulating electric current; (b),(c) ion densities for $\mathrm{KBr}$ and $\mathrm{HCl}$ respectively (solid and dashed lines are density contours for cations and anions, labeled by concentration).

I solve Eq. (4) in this square domain, with $\mathbf{I} \cdot \mathbf{n}=0$ on the boundaries (for details see Supplemental Material [39]). Figure 1(a) shows that there is a significant liquid junction potential $(\Delta \phi \approx 62 \mathrm{mV})$ between the two lower quadrants, corresponding approximately to the expected value for $\mathrm{HCl}$ treated as a binary electrolyte. Between the two upper quadrants, the junction potential is much weaker $(\Delta \phi \approx 25 \mathrm{mV})$ as might be expected in the presence of a supporting electrolyte [36]. It is essentially this difference that drives the circulating electric current [Fig. 1(b)]. By joining the upper and lower halves, it is as if we have shortcircuited the two liquid junctions, as sketched in Fig. 1(c). In the lower-left quadrant where the conductivity is small, this current corresponds to a significant Ohmic electric field as shown by the equipotential lines in Fig. 1(a). Also Eq. (3) implies I should be irrotational as well as solenoidal, in regions where the gradients vanish. This explains why approximately $|\mathbf{I}| \sim 1 / r$ in the lower left quadrant [Fig. 1(d)], and why the equipotential lines are approximately radial [Fig. 1(a)]. Finally, also shown in Fig. 1(a) is the space charge from $\rho_{z}=-\left(\epsilon k_{\mathrm{B}} T / e^{2}\right) \nabla^{2} \varphi$. Note that $\left|\rho_{z}\right| \lesssim 10^{-8} \mathrm{M}$ so that local charge neutrality should normally be a very good approximation [42].

The situation in Fig. 1 can only be transient since the initially localized crossed gradients relax by diffusion [43]. Thus I next solve the steady-state Nernst-Planck equations, preserving the 100-fold gradients by imposing fixed chemical potential boundary conditions for the relevant ion pairs: $\mathrm{HCl}$ on the left and right edges, and $\mathrm{KBr}$ on the top and bottom edges [44]. Such boundary conditions represent non-Faradaic sources and sinks, as detailed in the Supplemental Material [39]. Zero-flux boundary conditions are used otherwise, and this combination ensures that no current enters or leaves the domain $(\mathbf{I} \cdot \mathbf{n}=0)$.

Figure 2 shows features of the steady-state solution. First, note that a circulating current persists [Fig. 2(a)] albeit somewhat reduced compared to the initial situation. However this can no longer be cleanly mapped to an equivalent electric circuit since the region where $\nabla \sigma \times$ $\nabla g \neq 0$ now fills the domain. Second, the circulating current (which corresponds to cations moving clockwise and anions moving anticlockwise) distorts the ion density profiles [Figs. 2(b) and 2(c)]. Third, the more mobile $\mathrm{H}^{+}$ spreads out further than the less mobile $\mathrm{Cl}^{-}$[Fig. 2(c)] because the ion densities become increasingly decoupled with the increasing concentration of $\mathrm{KBr}$. Finally note that $\left[\mathrm{H}^{+}\right] \approx 0.3$ on the left-hand side. Bearing in mind the boundary condition [44], this implies an induced EDL with a wall potential $\frac{1}{2}\left(k_{\mathrm{B}} T / e\right) \ln \left(\left[\mathrm{Cl}^{-}\right] /\left[\mathrm{H}^{+}\right]\right) \approx-87 \mathrm{mV}$ [45]. This has potentially significant ramifications for diffusioosmosis as discussed below.

What are the implications for diffusiophoresis of a colloidal particle in such crossed salt gradients? By reverting Eq. (2), the diffusiophoretic drift velocity can be decomposed into a vector sum of three contributions [46],

$$
\mathbf{U} / \Gamma_{0}=4 \ln \cosh \left(\frac{1}{4} \bar{\zeta}\right) \nabla \ln \rho+\bar{\zeta} \nabla g / \sigma+\bar{\zeta} \mathbf{I} / \sigma
$$

In this $\rho=\sum_{i} \rho_{i}$ is the total ion density and $\bar{\zeta}=e \zeta / k_{\mathrm{B}} T$ is the nondimensionalized zeta potential. The overall scale is set by $\Gamma_{0}=(\epsilon / \eta)\left(k_{\mathrm{B}} T / e\right)^{2}$ where $\eta$ is the viscosity of the medium $\left(\Gamma_{0} \approx 510 \mu \mathrm{m}^{2} \mathrm{~s}^{-1}\right.$ in water at room temperature). In Eq. (5) the first term is chemiphoresis in the local ion gradients, the second is electrophoresis also arising from local gradients, and the third is a nonlocal electrophoretic contribution deriving from the field associated with the current. Equation (5) is appropriate to large (e.g., $\mu$ m-sized) particles and is derived by adapting the existing theory to the present situation [6,18]. For details see Supplemental Material [39].

Figure 3(a) illustrates this for a $\zeta=-40 \mathrm{mV}$ particle placed close to the left-hand edge of the square domain in Fig. 2 (i.e., in the steady-state solution of the Nernst-Planck equations). Of the three contributions, that from the current is the smallest, but nevertheless amounts to $\approx 20 \%$ of the resultant drift velocity. Overall, this nonlocal current contribution is largest near the edges of the square domain, and can contribute up to $20 \%-30 \%$ to the total magnitude of the drift [Fig. 3(b)]. Representative trajectories computed by integrating the kinematic equation $d \mathbf{R} / d t=\mathbf{U}$ are 


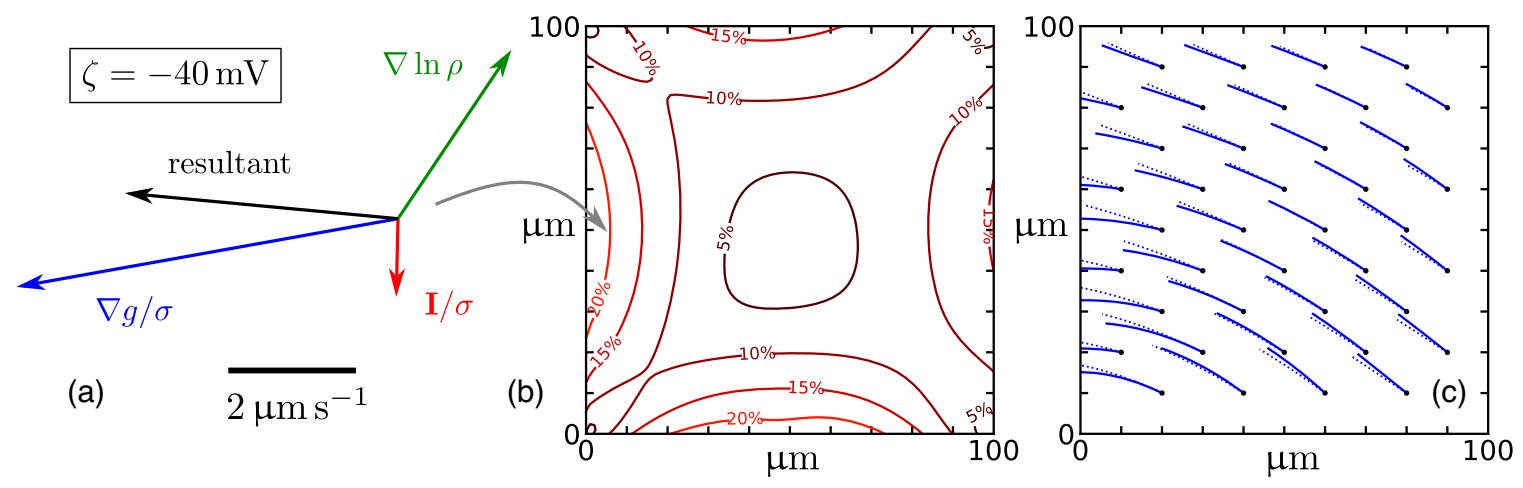

FIG. 3. Diffusiophoresis in steady-state crossed salt gradients. (a) Contributions to drift velocity (drawn to scale; prefactors omitted from labels) at the indicated point near the left-hand wall $(x=5 \mu \mathrm{m}, y=50 \mu \mathrm{m})$. (b) Ratio of magnitude of nonlocal current contribution to magnitude of resultant, as a percentage. (c) Particle trajectories from marked starting points (5 s duration; some reach the left-hand wall before the time is up; dotted lines are calculated omitting nonlocal contribution). All calculations are for $\zeta=-40 \mathrm{mV}$ (Fig. S2 in Supplemental Material shows results for other values of $\zeta$ ).

shown in Fig. 3(c). The current (circulating clockwise) causes negatively charged particles to drift downwards near the left-hand wall, where the effect is strongest since the conductivity $\sigma$ is smallest. Figure S2 in the Supplemental Material shows trajectories calculated for other values of the zeta potential. There is a considerable dependence on the sign and magnitude of the particle charge, indicative of the possibility to exploit the effect for particle sorting.

The calculated trajectories neglect a possible stirring of the fluid by diffusio-osmosis at the walls. The effect may be significant, since as we have seen the boundary conditions may induce large EDLs at the walls. Equation (5) can be repurposed for diffusio-osmotic "wall slip" if the sign is reversed and the gradients are projected parallel to the wall. In this case, assuming $\zeta \approx-90 \mathrm{mV}$ the slip could be as much as $4-5 \mu \mathrm{m} \mathrm{s}^{-1}$ (downwards) on the left-hand wall, with the nonlocal current contributing $\approx 40 \%$ of this. The domain would then resemble a (low-Reynolds number) lid-driven cavity, with the "lid" being the left-hand side. The particle trajectories could be significantly perturbed in the resulting flow [47]. Exploration of such fully coupled problems is left for future work.

The design of devices which exploit these striking effects is a clearly a promising avenue for future work. I note that in this situation one loses the logarithmic sensitivity exhibited in binary electrolytes $[2,15,48]$, so that in relying solely on transient gradients, the distance over which particles move is limited by the relaxation time for these gradients. This can be alleviated engineering steady-state gradients as in the present case study, using soluto-inertial beacons $[7,24]$ or other semipermanent sources and sinks, or by designing microfluidic devices in which long-lived gradients can be established [1,6,10].

To summarize, a rich phenomenology arises in the NernstPlanck equations when considering multicomponent electrolytes in more than one dimension. Circulating (solenoidal) electric currents appear when patches with different liquid junction potentials are connected by the intervening electrolyte solution, and the electric fields associated with these currents can make a significant nonlocal contribution to diffusiophoresis of suspended colloidal particles.

I thank Sangwoo Shin and Howard A. Stone for a critical reading of the draft manuscript. This work was partially supported by the STFC Hartree Centre's "Innovation: Return on Research" programme, funded by the Department for Business, Energy \& Industrial Strategy.

*patrick.warren@stfc.ac.uk

[1] B. Abécassis, C. Cottin-Bizonne, C. Ybert, A. Ajdari, and L. Bocquet, Nat. Mater. 7, 785 (2008).

[2] J. Palacci, C. Cottin-Bizonne, C. Ybert, and L. Bocquet, Soft Matter 8, 980 (2012).

[3] J. J. McDermott, A. Kar, M. Daher, S. Klara, G. Wang, A. Sen, and D. Velegol, Langmuir 28, 15491 (2012).

[4] A. Reinmüller, H. J. Schöpe, and T. Palberg, Langmuir 29, 1738 (2013).

[5] D. Florea, S. Musa, J. M. Huyghe, and H. M. Wyss, Proc. Natl. Acad. Sci. U.S.A. 111, 6554 (2014).

[6] N. Shi, R. Nery-Azevedo, A. I. Abdel-Fattah, and T. M. Squires, Phys. Rev. Lett. 117, 258001 (2016).

[7] A. Banerjee, I. Williams, R. N. Azevedo, M. E. Helgeson, and T. M. Squires, Proc. Natl. Acad. Sci. U.S.A. 113, 8612 (2016).

[8] H. J. Keh, Curr. Opin. Colloid Interface Sci. 24, 13 (2016).

[9] D. Velegol, A. Garg, R. Guha, A. Kara, and M. Kumara, Soft Matter 12, 4686 (2016).

[10] S. Shin, O. Shardt, P. B. Warren, and H. A. Stone, Nat. Commun. 8, 15181 (2017).

[11] S. Shin, J. T. Ault, P. B. Warren, and H. A. Stone, Phys. Rev. X 7, 041038 (2017).

[12] R. P. Sear, Phys. Rev. Lett. 122, 128101 (2019).

[13] A. Kar, T.-Y. Chiang, I. O. Rivera, A. Sen, and D. Velegol, ACS Nano 9, 746 (2015). 
[14] S. Shin, E. Um, B. Sabass, J. T. Ault, M. Rahimi, P. B. Warren, and H. A. Stone, Proc. Natl. Acad. Sci. U.S.A. 113, 257 (2016).

[15] S. Shin, P. B. Warren, and H. A. Stone, Phys. Rev. Applied 9, 034012 (2018).

[16] S. Shin, J. T. Ault, J. Feng, P. B. Warren, and H. A. Stone, Adv. Mater. 29, 1701516 (2017).

[17] H. Lee, J. Kim, J. Yang, S. W. Seo, and S. J. Kim, Lab Chip 18, 1713 (2018).

[18] J. L. Anderson, Annu. Rev. Fluid Mech. 21, 61 (1989).

[19] A. Brown and W. Poon, Soft Matter 10, 4016 (2014).

[20] T.-Y. Chiang and D. Velegol, J. Colloid Interface Sci. 424, 120 (2014).

[21] A. Gupta, S. Shim, L. Issah, C. McKenzie, and H. A. Stone, Soft Matter 15, 9965 (2019).

[22] A. Gupta, B. Rallabandi, and H. A. Stone, Phys. Rev. Fluids 4, 043702 (2019).

[23] J. L. Wilson, S. Shim, Y. E. Yu, A. Gupta, and H. A. Stone, Langmuir (2020), https://doi.org/10.1021/acs .langmuir.9b03333.

[24] A. Banerjee and T. M. Squires, Sci. Adv. 5, eaax1893 (2019).

[25] V. G. Levich, Physicochemical Hydrodynamics (PrenticeHall, Englewood Cliffs, NJ, 1962).

[26] J. Newman and K.E. Thomas-Alyea, Electrochemical Systems (John Wiley \& Sons, Hoboken, NJ, 2004).

[27] D. R. Hafemann, J. Phys. Chem. 69, 4226 (1965).

[28] H. J. Hickman, Chem. Eng. Sci. 25, 381 (1970).

[29] J. L. Jackson, J. Phys. Chem. 78, 2060 (1974).

[30] V. M. Aguilella, S. Mafé, and J. Pellicer, Electrochim. Acta 32, 483 (1987).

[31] M. Z. Bazant, K. Thornton, and A. Ajdari, Phys. Rev. E 70, 021506 (2004).

[32] M. Janssen and M. Bier, Phys. Rev. E 97, 052616 (2018).

[33] The value of $\varphi$ where the outer solution meets the EDLs cannot be predetermined, and indeed it is the mismatch between this and the true wall boundary condition that gives rise to an EDL in the first place.

[34] Technically I also assume small Dhukin number so that surface conduction in the EDLs can be neglected [18].

[35] In a multiply connected domain one can have $\mathbf{I} \neq 0$ even if there are no explicit crossed gradients. An example would be the annulus created by excising the central region of the square domain in the case study in Fig. 1.

[36] The binary electrolyte case can be solved because the individual ion densities are slaved to each other by local charge neutrality so that $g=q_{1} q_{2}\left(D_{1}-D_{2}\right) \rho_{s}$ and $\sigma=$ $q_{1} q_{2}\left(q_{1} D_{1}+q_{2} D_{2}\right) \rho_{s}$. Hence $g=\beta \sigma$ and $\mathbf{E}=\sigma^{-1} \nabla g$ can be integrated to determine $\varphi$ up to a a constant. A uniform background can also be accommodated, since in that case also $\nabla g=\beta \nabla \sigma$ and therefore $\varphi=-\beta \ln \sigma$.

[37] R. A. Rica and M. Z. Bazant, Phys. Fluids 22, 112109 (2010).
[38] C. A. Coulson, Electricity (Oliver and Boyd, Edinburgh, 1961).

[39] See Supplemental Material at http://link.aps.org/ supplemental/10.1103/PhysRevLett.124.248004 which contains a uniqueness theorem for Eq. (4) and remarks on the equivalent variational principle thereof, details of the constant chemical potential boundary conditions, and the numerical solution of Eqs. (1)-(4), and a derivation of the diffusiophoretic drift velocity in Eq. (5).

[40] The diffusivities of $\mathrm{H}^{+}, \mathrm{Cl}^{-}, \mathrm{K}^{+}$, and $\mathrm{Br}^{-}$were taken as $9.31,2.03,1.96$, and $2.01 \times 10^{-9} \mathrm{~m}^{2} \mathrm{~s}^{-1}$ respectively, from a database in the PHREEQC software package; see D. L. Parkhurst and C. A. J. Appelo, Description of input and examples for PHREEQC version 3: A computer program for speciation, batch-reaction, one-dimensional transport, and inverse geochemical calculations, U.S. Geological Survey Techniques and Methods, book 6, Chap. A43, 497 p., available only at https://pubs.usgs.gov/tm/06/a43/.

[41] The specific functional form is $c=c_{0}+\left(c_{1}-c_{0}\right) \operatorname{erf}(x / w)$ where $c_{0}=0.01$ and $c_{1}=1$ are the limiting concentrations in arbitrary units, $x$ is the distance from the midplane, and $w=10 \mu \mathrm{m}$ is the width.

[42] Interestingly the choice of units for the ion densities does not affect the calculation of the space charge $\rho_{z}$.

[43] The ion densities relax on a timescale $O\left(L^{2} / D\right) \sim 10 \mathrm{~s}$ since $L=100 \mu \mathrm{m}$ and $D \sim 10^{-9} \mathrm{~m}^{2} \mathrm{~s}^{-1}$. On the other hand, the time scale for the electrostatic potential $\varphi$ and electric current $\mathbf{I}$ to relax is $O\left(\lambda_{D}^{2} / D\right)$ [28,29] [as an $R C$ circuit, the relevant capacitance is $O\left(\lambda_{D} / L\right)$ smaller than in the EDL charging problem [31,32] ]. Then $L \gg \lambda_{D}$ implies $\varphi$ and $\mathbf{I}$ are slaved to the ion densities.

[44] Constant chemical potential boundary conditions correspond to injecting a neutral flux combination $J_{+}=J_{-}$such that the product $\rho_{+} \rho_{-}$is fixed at the boundary. Typically, this requires solving a quadratic equation for each ion pair, at each boundary node and each time step. For the present case, I impose $\left[\mathrm{H}^{+}\right]\left[\mathrm{Cl}^{-}\right]=10^{-4}$ and 1 on the left and right edges, respectively, and $\left[\mathrm{K}^{+}\right]\left[\mathrm{Br}^{-}\right]=10^{-4}$ and 1 on the bottom and top edges, respectively. For more details, see Supplemental Material [39].

[45] This is referenced to the state where $\left[\mathrm{H}^{+}\right]=\left[\mathrm{Cl}^{-}\right]$ which for simplicity I assume is the "inner" boundary condition.

[46] Equation (5) assumes the ions are univalent [22,23].

[47] The ion densities themselves could be perturbed if the Peclet number $U L / D$ for ion transport becomes $O(1)$. It then becomes necessary to solve the Nernst-Planck equations with a convective term, together with Eq. (4) for the potential, and Stokes's equation for the flow.

[48] Logarithmic sensitivity in binary electrolytes follows by inserting $\rho=2 \rho_{s}, \sigma^{-1} \nabla g=\beta \nabla \ln \rho_{s}$ (from [36]) and $\mathbf{I}=0$ into Eq. (5), whereupon $\mathbf{U} \propto \nabla \ln \rho_{s}$. 